\title{
XEUS: the physics of the hot evolving universe
}

Monique Arnaud - Xavier Barcons • Didier Barret • Marshall Bautz • Ronaldo Bellazzini - Johan Bleeker · Hans Böhringer · Thomas Boller • William Nielsen Brandt • Massimo Cappi • Francisco Carrera • Andrea Comastri - Enrico Costa • Thierry Courvoisier • Piet de Korte · Tom Dwelly • Andrew Fabian · Kathryn Flanagan · Roberto Gilli · Richard Griffiths • Günther Hasinger • Jelle Kaastra • Steve Kahn • Richard Kelley • Hideyo Kunieda - Kazuo Makishima • Giorgio Matt • Mariano Mendez • Kazuhisa Mitsuda • Kirpal Nandra • Takaya Ohashi · Mathew Page • Giorgio Palumbo • Mikhail Pavlinsky • Salvatore Sciortino • Alan Smith · Lothar Strüder · Tadayuki Takahashi • Marc Türler • Martin Turner • Yoshihiro Ueda • Cristian Vignali • Jacco Vink · Robert Warwick • Mike Watson · Richard Willingale · Shuang Nan Zhang

Received: 21 March 2008 / Accepted: 27 May 2008 / Published online: 30 July 2008 (C) Springer Science + Business Media B.V. 2008

M. Arnaud

CEA/DSM/DAPNIA/SAP, CEA-Saclay, Gif-sur-Yvette, France

X. Barcons · F. Carrera

IFCA(CSIC-UC), Santander, Spain

D. Barret

CESR, CNRS/UPS, Toulouse, France

M. Bautz

Massachusetts Institute of Technology, Cambridge, MA, USA

R. Bellazzini

INFN, Pisa, Italy

J. Bleeker · P. de Korte · J. Kaastra

SRON/U. Utrecht, Utrecht, Netherlands

H. Böhringer · T. Boller · G. Hasinger · L. Strüder

MPE, Garching, Germany

W. N. Brandt

Pennsylvania State University, University Park, PA, USA

M. Cappi

INAF/IASF, Bologna, Italy

A. Comastri · R. Gilli (凶)

INAF/Oss. Astron. Bologna, Bologna, Italy

e-mail: roberto.gilli@oabo.inaf.it 
Abstract This paper describes the next generation X-ray observatory XEUS which has been submitted to the European Space Agency in the framework of the Cosmic Vision 2015-2025 competition and has been selected for an assessment study. The paper summarizes the scientific goals and instrumental concepts of the proposed X-ray telescope with $5 \mathrm{~m}^{2}$ effective area and angular resolution better than 5 arc sec.

Keywords XEUS • Cosmic vision $\cdot$ X-ray astronomy

\section{Introduction}

The X-ray Evolving Universe Spectroscopy mission (XEUS) is Europe's next generation X-ray observatory. With unprecedented sensitivity to the hot,

E. Costa

INAF/IASF, Rome, Italy

T. Courvoisier · M. Türler

ISDC, Geneva, Switzerland

T. Dwelly

University of Southampton, UK

\section{A. Fabian}

Institute of Astronomy, Cambridge, UK

K. Flanagan

STScI, Baltimore, USA

R. Griffiths

Carnegie Mellon University, Pittsburgh, USA

S. Kahn

KIPAC, SLAC, Stanford University, USA

R. Kelley

NASA GSFC, Greenbelt, USA

H. Kunieda

Nagoya University, Nagoya, Japan

K. Makishima

University of Tokyo, Tokyo, Japan

G. Matt

U. Roma Tre, Rome, Italy

M. Mendez

Kapteyn Astronomical Institute, University of Groningen,

Groningen, Netherlands 
million-degree Universe, XEUS will provide the long-sought answers to many of the key questions in contemporary astrophysics:

- How did supermassive black holes form and grow and how did they influence galaxy-growth?

- How did large scale structure evolve and how was the baryonic component chemically enriched?

- How does matter behave under the influence of strong gravity?

XEUS will constitute a cornerstone element in the "dual track" approach towards the study of structure formation, which requires us to understand simultaneously the evolution of the hot and cold components of the Universe. In this sense, XEUS is fully complementary to the major future ground- and space-based observatories, JWST, LISA, ALMA, ELT and SKA. X-rays can penetrate through obscuring gas and dust in the centres of young galaxies and so can disentangle the ambiguity between star-formation and accretion power in the evolving Universe. Dark matter, which can currently only be studied through its gravitational action on visible matter, can be probed through

K. Mitsuda · T. Takahashi

ISAS/JAXA, Sagamihara, Japan

K. Nandra

Imperial College, London, UK

T. Ohashi

Tokyo Metropolitan University, Tokyo, Japan

M. Page $\cdot$ A. Smith

MSSL/UCL, Holmbury St. Mary, Dorking, UK

G. Palumbo · C. Vignali

University of Bologna, Bologna, Italy

M. Pavlinsky

IKI, Moscow, Russia

S. Sciortino

INAF/Oss. Astron., Palermo, Italy

M. Turner · R. Warwick · M. Watson · R. Willingale

University of Leicester, Leicester, UK

Y. Ueda

University of Kyoto, Kyoto, Japan

J. Vink

University of Utrecht, Netherlands

S. N. Zhang

IHEP, Tsinghua University, Beijing, China 
the evolution of large-scale structures, traced by the hot X-ray-emitting gas trapped in the dark matter potential wells. While significant progress has been made in constraining the cosmological parameters and in reconstructing the large-scale structure of the dark matter distribution, we still lack even a basic understanding of the spatial and chemical evolution of the baryonic component of the Universe. XEUS is needed both to observe directly the evolution of the warm/hot baryonic matter in the intergalactic medium and the hot plasma in galaxy clusters, and to trace galaxy evolution through the effects of energy release and nucleosynthesis in the intergalactic medium. The most extreme physical conditions in the Universe, i.e. regions with the strongest gravity, the highest densities, the hottest temperatures and the largest magnetic fields, occur in the immediate vicinity of black holes and in neutron stars. Since X-rays constitute the major component of radiation from the innermost parts of the accretion flow and from compact surfaces, XEUS can address how gravity works in the strong field limit, and the equation of state of matter at (supra)nuclear densities.

XEUS will be placed in a halo orbit around L2, by a single Ariane 5 ECA, and comprises two spacecraft. The optics assembly of XEUS is contained in the Mirror Spacecraft (MSC) while a suite of five focal plane instruments is contained in the Detector Spacecraft (DSC), which is maintained at the focus of the mirror by formation flying. L2 provides the necessary benign gravity gradient environment for formation flying and also allows efficient thermal control of the optics and instruments, and long continuous observations. The main requirement for XEUS is to provide a focused beam of X-rays with an effective aperture of $5 \mathrm{~m}^{2}$ at $1 \mathrm{keV}$ and a spatial resolution better than 5 arc sec half-energy width. This is achieved using silicon micro-pore optics, which gives an advantage, in terms of mass/unit collecting area, of more than an order of magnitude compared to the largest current X-ray telescope, aboard XMM-Newton. In the current XEUS baseline there are two major focal plane instruments. The narrow-field instrument (NFI) comprises a cryogenic array of superconducting Transition Edge Sensors to give energy resolution of 2 to $6 \mathrm{eV}$ FWHM over the energy range $0.1-8 \mathrm{keV}$, combined with imaging over a field of view $(\mathrm{FoV})$ of $1.6 \times 1.6$ arc min square. The Wide Field Imager (WFI), based on silicon pixel arrays, has a FoV of $7 \times 7$ arc min (goal $10 \times$ 10 arc min) square, with 30 to $150 \mathrm{eV}$ energy resolution. These two instruments are supplemented with three smaller instruments, which are needed to address specific science aims: The Hard X-ray Imager (HXI); the High Time Resolution Spectrometer (HTRS) and the X-ray POLarimeter (XPOL).

\section{The physics of the hot, evolving universe}

Astrophysics and Cosmology are currently in a Golden Age. Precision measurements have determined the cosmic geometry and history. After an early inflationary phase dark energy, dark matter and hot baryons emerge as the 
three dominating forms of matter and energy density in the contemporary Universe. An indepth and coherent investigation of the formation and evolution of structure in the Universe will require a highly sensitive "dual track" observational approach, studying both the cold baryonic matter and, in a fully complementary manner, the evolution of hot baryonic matter. The formation of structure from cold matter on the stellar/galaxy mass scale can be probed at infrared and (sub)millimeter wavelengths by e.g. Herschel, JWST and ALMA. $\mathrm{X}$-ray observations are uniquely suited to study the warm/hot baryons embedded in the dark matter potential wells of the cosmic web, and to understanding the evolution and extreme physics of accretion powered sources.

\subsection{Co-evolution of galaxies and their supermassive black holes}

It has become increasingly apparent that the formation of supermassive black holes (SMBH) in Active Galactic Nuclei (AGN) is an integral feature of galaxy evolution. Theory, observations and numerical N-body simulations all suggest that AGN may be the crucial link responsible for regulation of star formation in massive galaxies, but the details of these processes are as yet completely unclear, due to lack of quantitative information. A key goal of XEUS is therefore to study the evolving violent Universe by tracing mass accretion onto black holes through cosmic time out to the highest possible redshifts.

- The first black holes: XEUS is uniquely configured to discover and study the early growth phase of the first black holes, which are rendered invisible in other wavebands due to intergalactic absorption and dilution by their host galaxy. To detect X-ray emitting black holes out to $z>10$ and to investigate their growth requires an unprecedented combination of large throughput, high angular resolution and large FoV in the X-ray band. The expected X-ray flux of a $10^{6} \mathrm{M}_{\odot} \mathrm{SMBH}$ accreting at its Eddington limit at $z=10$ is $3 \times 10^{-18} \mathrm{erg} \mathrm{cm}^{-2} \mathrm{~s}^{-1}$. This is the main science driver for the large effective area at $1 \mathrm{keV}$ and sets the angular resolution for the XEUS mission to better than 5 arc sec.

- Obscured black hole growth: The deepest Chandra and XMM-Newton surveys miss a significant fraction of the total AGN population, with at least $50 \%$ of the $>6 \mathrm{keV}$ background still unresolved [1] and probably due to heavily obscured, Compton-thick AGNs predicted in population synthesis models [2]. By combining the capabilities of the XEUS HXI and WFI detectors, an almost complete census of the physical properties of these objects will become possible over $90 \%$ of cosmic time. Figure 1 shows a simulation of a deep survey, which clearly indicates XEUS's ability to detect and characterize the important source classes throughout the whole redshift range.

- Feedback and downsizing: The most promising explanation for the correlation between black hole mass and host galaxy properties is that massive outflows from AGN terminate the growth of stellar and black hole 

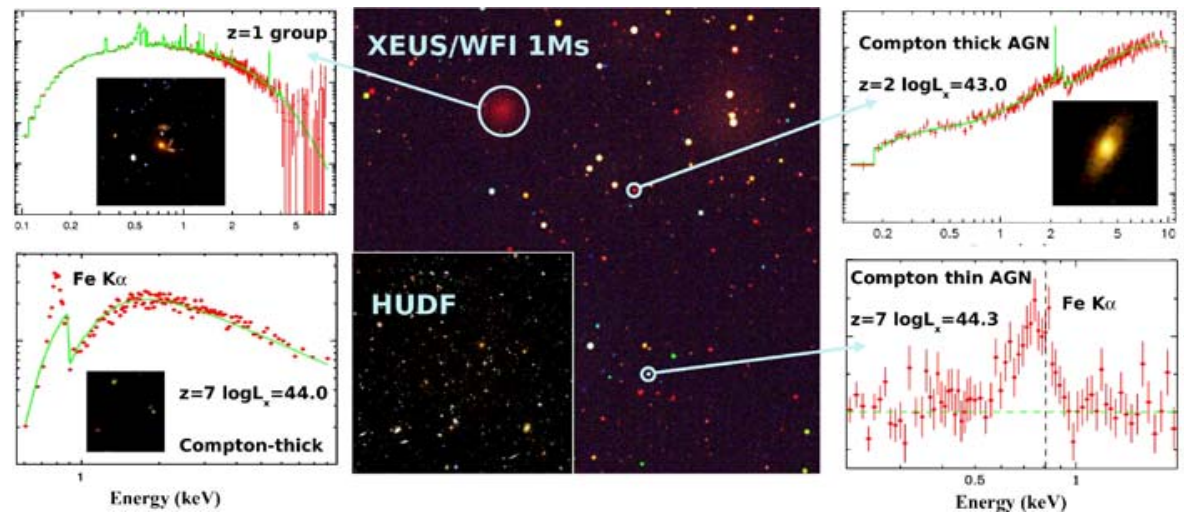

Fig. 1 Simulation of a 1 Ms XEUS WFI observation of the Chandra Deep Field South (CDFS). We assume a PSF HEW of $2^{\prime \prime}$. Real CDFS sources have been augmented with fainter, absorbed AGN based on the latest X-ray background synthesis model [2], and a population of starburst galaxies based on the GOODS/Spitzer $24 \mu \mathrm{m}$ data. The HST ACS Ultradeep Field, the deepest optical image so far, is shown as an insert. To the left and right are X-ray spectra for different objects from the same simulation. The upper left shows the spectrum ( $v \mathrm{~F}_{v}$ space, arbitrary units) of a known high redshift $(z \sim 1)$ galaxy group. The upper right shows a $z=2$ Compton-thick object with $\mathrm{L}_{X}=10^{43} \mathrm{erg} \mathrm{s}^{-1}$. The HST image (inset) shows a bulge galaxy-these kinds of AGN may be optically invisible but can dominate the obscured accretion budget of the Universe. Slightly more luminous examples can be detected by XEUS, and their redshifts determined solely from the X-ray spectra, out to $z=7$ (bottom left). At the bottom right we show a data/model ratio for a type-1 (unobscured) AGN at the same redshift with $\mathrm{L}_{X}=2 \times 10^{44} \mathrm{erg} \mathrm{s}^{-1}$. We simulate a narrow $\mathrm{Fe}-\mathrm{K}_{\alpha}$ emission line with a rest frame equivalent width of $200 \mathrm{eV}$ plus an accretion disk line with parameters similar to MCG6-30-15 $(\mathrm{EW}=300 \mathrm{eV})$. Though challenging even for XEUS, there is the prospect that we might measure relativistic effects in the iron lines of individual AGN out to the dark ages of the Universe

components by driving the interstellar medium out into intergalactic space [3]. XEUS will have the sensitivity and energy resolution not only to estimate the density and location of the outflows by studying time variability in nearby objects, but more importantly will characterize winds in "typical" QSOs at $z=1-3$, where the majority of galaxy growth occurs.

- X-ray redshifts: a new tool to characterise the universe: Traditionally, X-ray surveys have relied upon painstaking followup work in other wavebands to determine redshifts and clarify the nature of the detected sources. The combined sensitivity, spectral resolution and bandpass of XEUS will, for the first time, allow source classification and redshift determination autonomously, based on the X-ray data alone.

\subsection{Evolution of large scale structure and nucleosynthesis}

The large scale structure of today's Universe is determined by the growth of dark matter density fluctuations, and by the dynamical action of dark matter and dark energy. Its appearance is determined by the visible, baryonic matter 
embedded in the dark matter distribution, but there are still major gaps in our understanding of the baryonic component.

XEUS, combining very large collecting power with excellent energy resolution and good spatial resolution, is needed to directly observe the evolution of the baryonic matter in the warm/hot intergalactic medium (WHIM) and the hot plasma in galaxy clusters, and to trace galaxy evolution through the effects of energy release and nucleosynthesis.

- The missing baryons: While baryons determine the visible appearance of the present Universe, about half of the baryons in the local Universe are unidentified, presumably constituting the WHIM with temperatures of $10^{5-7} \mathrm{~K}$. This gas, photoionized by massive stars and active galaxies, has received additional heating during the formation of large-scale filaments, and has been enriched by heavy elements due to matter expelled from galaxies in early star-formation epochs [4]. The photon collecting power of XEUS will be 300 times higher than that of the high-resolution spectrometers on Chandra and XMM-Newton, which have made tentative WHIM detections already $[5,6]$.

- The physics of the first clusters: Feedback from supernovae and SMBH must play a significant role, not only in the evolution of individual galaxies, but also in groups and clusters as a whole. These feedback mechanisms are likely to provide the extra energy required to keep cluster centres from cooling all the way down to molecular clouds, to account for the entropy excess observed in the gas of groups and clusters, and to cure the overcooling problem and regulate star formation [7]. XEUS can perform detailed studies of the first groups and clusters in the range $z=1.5-2$ (Fig. 2). Temperature, entropy and mass profiles will be measured for low mass systems up to $z=1$, with a precision currently achieved only for bright local objects.

- The mass distribution of evolving clusters: the cluster mass and the dynamical structure are key properties in the use of galaxy clusters as cosmological probes and as laboratories for the cosmic evolution of the baryonic component. Present cluster mass determinations are hampered by (1) the inability to distinguish a mixture of temperatures from a single temperature, leading to significant errors in the mass determination, and (2) our ignorance of the dynamical pressure of gas motions in the cluster intracluster medium. Both problems can be tackled by XEUS high-resolution NFI spectroscopy in regions of nearby clusters and in deeper exposures of distant clusters (Fig. 2).

\subsection{Matter under extreme conditions}

The most extreme physical conditions in the observable Universe occur around black holes and neutron stars. Here, the most dramatic events and powerful sources can be used to test physics and astrophysics to the limit. Testing our understanding of General Relativity (GR) requires observations just 

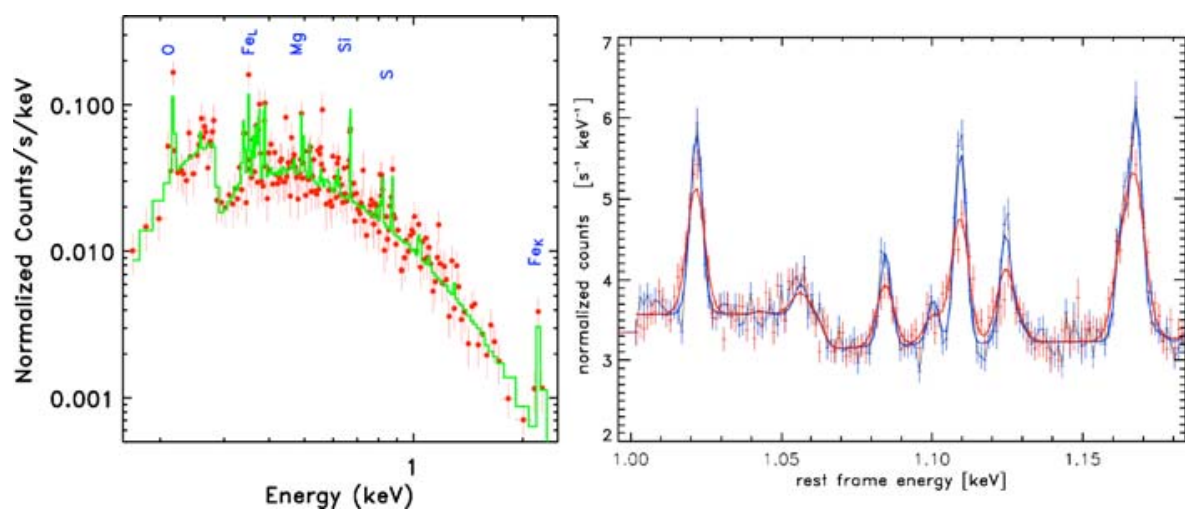

Fig. 2 Left: XEUS NFI $150 \mathrm{ks}$ observation of a $\mathrm{kT}=2 \mathrm{keV}, z=2$ cluster with a bolometric luminosity of $7.7 \times 10^{43} \mathrm{erg} \mathrm{s}^{-1}$. The overall temperature and element abundances can be measured accurately: $\pm 2.8 \%$ for $\mathrm{kT}, \pm 30 \%$ for $\mathrm{O}$ and $\mathrm{Mg}, \pm 18 \%$ for $\mathrm{Si}$ and $\pm 11 \%$ for $\mathrm{Fe}$. Temperature and abundance profiles can be measured easily out to at least $z \sim 1$. Right: XEUS NFI spectra around the Fe L-line complex for a cluster with $\mathrm{F}_{X}=1.5 \times 10^{-14} \mathrm{erg} \mathrm{cm}^{-2} \mathrm{~s}^{-1}$ at $z=1$. Velocity broadening by 100 (blue) and $600 \mathrm{~km} \mathrm{~s}^{-1}$ (red) are assumed, and can be determined to within $20-50 \mathrm{~km} \mathrm{~s}^{-1}$ in a $250 \mathrm{ks}$ observation

outside the event horizon of black holes, where extreme gravity produces large Doppler shifts, gravitational redshifts and light bending, as well as frame dragging if the central mass is rotating. Studies of the accretion flow around and upon the surfaces of neutron stars provide constraints on the equation of state (EoS) of nuclear matter and therefore on the strong interaction. All these effects can be seen or inferred from the X-ray spectra and variability of accreting black holes and neutron stars.

- Gravity in the strong field limit: the accretion flow in luminous sources consists of an optically thick accretion disc with a Comptonizing coronal region above and below it. The corona irradiates the disc giving rise to a reflection spectrum, consisting of backscattered continuum with superposed fluorescent and recombination lines. Close to the black hole, the whole reflection spectrum is relativistically broadened. Measurements of the degree of broadening then translate into values of the innermost radius of the disc and thus, via the dependence of the innermost stable orbit on the angular momentum of the black hole, into the determination of the black hole spin. The flow is turbulent, so transient orbiting blobs are expected [8]. Mapping their behaviour and measuring orbits over a range of radii will probe GR in the extreme regime. Complementary information can be obtained by polarimetric measurements, since the polarization properties of the emitted radiation are strongly modified by GR effects. The huge collecting area of XEUS will open up this crucial area for detailed study. The high spectral resolution of the NFI can trivially deconvolve narrow components, as well as a complete determination of the effects of complex absorption. Several hundred bright, nearby AGN can 
thereby be studied on timescales comparable to the inner orbital times, enabling the behaviour at different black hole masses and accretion rates to be explored. XEUS is also capable of extending such studies to the highest redshifts (Fig. 3 left).

- Extreme physics: In Neutron Stars (NSs) the density in the core can be several times nuclear, and the magnetic fields can exceed by ten orders of magnitude the strongest fields generated in terrestrial laboratories. Hyperon-dominated matter, deconfined quark matter, superfluidity, even superconductivity are predicted. Similarly, quantum electrodynamics predicts that in strong magnetic fields the vacuum becomes birefringent. This makes NSs ideal laboratories not only for astrophysics, but also for nuclear and particle physics. Different EoS predict different maximum masses and mass-radius relations. All mass measures cluster around the canonical $1.4 \mathrm{M}_{\odot}$, a value which can be accommodated by virtually all EoS. XEUS, by combining fast timing (HTRS) and improved spectral capabilities in X-rays (WFI, HTRS and NFI) will provide new probes of NS radii (see Fig. 3 right) to constrain the EoS. These diagnostics include (1) X-ray burst spectroscopy, enabling us to detect gravitationally redshifted emission lines and absorption edges, (2) waveform fitting of X-ray pulsations produced by rotating hot spots, either during X-ray bursts or in the persistent emission of pulsars, (3) X-ray spectroscopy of cooling
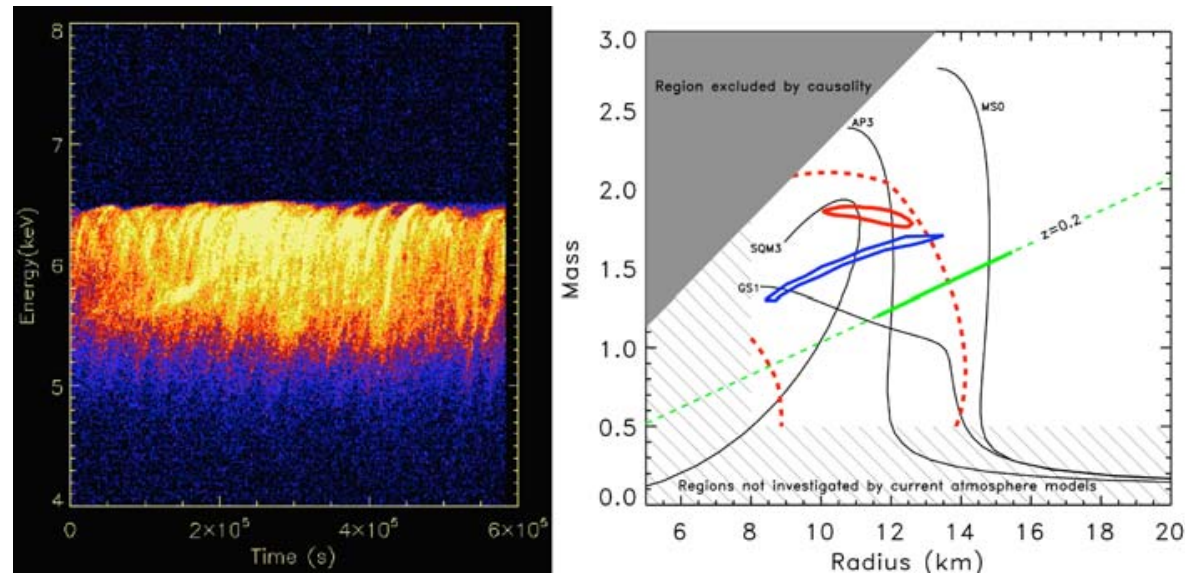

Fig. 3 Left: Excess iron line emission versus time for a simulated XEUS observation of iron lines from a turbulent disc around a black hole typical of a local AGN [8]. Right: Mass-radius relations for representative EoS involving standard nucleonic matter ( $A P 3$ and $M S O)$, strange quark matter $(S Q M 3)$, Kaon condensates (GS1). For illustrative purposes, the constraints derived from XEUS are from (1) a gravitational redshift $(z=0.2)$ for a source for which mass function is known (green bold solid and dashed lines), (2) waveform fitting of pulsations obtained from a $2 \mathrm{~h}$ observation of an accreting millisecond pulsar (blue contours), (3) hydrogen atmosphere model fitting of the X-ray spectrum of a quiescent NS in the globular cluster Omega Cen (red contours, 99\%). The best available constraints from XMM-Newton to date for the same object are also shown (red dashed lines) 
NSs and measurements of the associated cooling curves whose shape depends on the NS structure and internal composition, (4) the study of the sub-ms variability from the innermost regions of accretion disks (high frequency quasi-periodic oscillations, QPOs, recently discovered by the Rossi X-ray Timing Explorer, RXTE) and (5) the detection of kilohertz seismic vibrations in magnetars after giant flares.

\subsection{XEUS as an observatory}

In addition to its core science, XEUS has unique role as an observatory with a wide-ranging appeal and major impact on a vast array of science topics. To mention two out of many examples: (1) Hot cosmic plasmas emit mostly at X-ray wavelengths and XEUS will provide a unique opportunity to study their dynamics and chemical abundances in star forming regions, stars, supernovae and supernova remnants, as well as the interactions between these objects and their environments; (2) Sgr A*, the SMBH at the Galactic centre, shows an apparent X-ray reflection nebula which might indicate that the black hole was active in the very recent past. XEUS can definitively test, and further illuminate, this fascinating hypothesis via polarimetry.

\subsection{Requirements on the proposed payload}

To meet the science aims discussed above, a suite of focal plane instruments in conjunction with a high-throughput grazing incidence X-ray mirror system is proposed. The requirements imposed on this instrument complement are summarized in Table 1.

\section{Mission profile}

XEUS comprises both a MSC and a DSC. The MSC contains the X-ray optics and the DSC the prime focal plane instruments. The instruments, one at a time, will be kept at the primary focus of the $35 \mathrm{~m}$ focal length X-ray optics using the concept of formation flying.

\subsection{Launcher requirements}

The two spacecraft will be launched together in a single stack on the Ariane 5 ECA. The XEUS MSC, containing the mirror with its outer diameter of $4.2 \mathrm{~m}$, fits in the $4.57 \mathrm{~m}$ diameter of the Ariane 5 ECA fairing. The DSC will be mounted on the top of the MSC. With non-deployable baffles, the spacecraft stack fits in the length of the medium fairing (Fig. 4). In this configuration the launcher can place both spacecraft at L2 using a single launch for a total wet mass of 6.5 tonnes. 
Table 1 Science requirements on the payload

\begin{tabular}{|c|c|c|}
\hline Parameter & Requirement (goal) & Science driver \\
\hline$\overline{\text { Effective area }\left(\mathrm{m}^{2}\right)}$ & $\begin{array}{l}1(1.5) \text { at } 0.2 \mathrm{keV} \\
5 \text { at } 1 \mathrm{keV} \\
2 \text { at } 7 \mathrm{keV} \\
1 \text { at } 10 \mathrm{keV} \\
(0.1) \text { at } 30 \mathrm{keV}\end{array}$ & $\begin{array}{l}\text { WHIM, early BHs, clusters } \\
\text { Clusters, WHIM, early BHs } \\
\text { EOS, gravity in strong fields } \\
\text { EOS, acceleration, early BHs } \\
\text { Acceleration, early BHs, EOS }\end{array}$ \\
\hline Energy range (keV) & $0.1-40$ & BHs, acceleration, clusters \\
\hline Angular resolution $(\operatorname{arcsec})$ & $\begin{array}{l}5(2) \text { at }<10 \mathrm{keV} \\
10 \text { at } 40 \mathrm{keV}\end{array}$ & $\begin{array}{l}\text { Clusters, early BHs, WHIM } \\
\text { Early BHs }\end{array}$ \\
\hline \multirow[t]{2}{*}{ Field of view (arc min) } & $7 \times 7(10 \times 10):$ WFI, HXI & $\begin{array}{l}\text { Clusters, early BHs, } \\
\text { acceleration }\end{array}$ \\
\hline & $1.6 \times 1.6: \mathrm{NFI}$ & $\begin{array}{l}\text { Clusters, enrichment, } \\
\text { galaxy evolution }\end{array}$ \\
\hline \multirow[t]{4}{*}{ Spectral resolution (eV; FWHM) } & $2(1)$ at $0.5 \mathrm{keV}: \mathrm{NFI}$ & WHIM \\
\hline & $\begin{array}{l}2 \text { at }<2 \mathrm{keV}: \mathrm{NFI} \\
6(3) \text { at } 6 \mathrm{keV}: \mathrm{NFI}\end{array}$ & $\begin{array}{l}\text { Clusters } \\
\text { Clusters, enrichment, } \\
\text { galaxy evolution }\end{array}$ \\
\hline & 150 at $6 \mathrm{keV}:$ WFI & Early BHs \\
\hline & 1000 at $40 \mathrm{keV}: \mathrm{HXI}$ & Early BHs \\
\hline $\begin{array}{l}\text { Point source detection } \\
\text { sensitivity, erg cm }{ }^{-2} \mathrm{~s}^{-1}\end{array}$ & $\left(3 \times 10^{-18}\right)$ at $0.2-8 \mathrm{keV} ; 4 \sigma$ & Early BHs \\
\hline Time Resolution (s) & $10^{-5}:$ HTRS & EOS studies \\
\hline Count rate capability $\left(\mathrm{s}^{-1}\right)$ & $210^{6}:$ HTRS & EOS studies \\
\hline $\begin{array}{l}\text { Polarimetry (MDP, } \\
3 \sigma \text {-confidence in } 10 \mathrm{ks} \text { ) }\end{array}$ & $2 \%$ at $10^{-2}$ Crab: XPOL & EOS studies \\
\hline Observing constraints & $\begin{array}{l}>2 \text { weeks visibility each } 6 \mathrm{~m} \\
\text { ToO response in }(<1 \text { day }) \\
10^{3}\left(5 \times 10^{4}\right) \text { s cont. observ. } \\
\pm 5^{\circ}\left( \pm 15^{\circ}\right) \text { range Sun angle }\end{array}$ & $\begin{array}{l}\text { EOS studies } \\
\text { EOS studies } \\
\text { EOS studies, strong gravity } \\
\text { EOS studies }\end{array}$ \\
\hline
\end{tabular}

\subsection{Orbit requirements}

A Halo orbit around the second Lagrangian point of the Sun-Earth system (L2) is selected as providing optimal conditions with respect to perturbations, stable thermal environment, lack of eclipses, and sky visibility. The orbit is eclipse-free for $5^{+}$years, and can be reached by free-transfer in about 1 month for an almost full-year launch window. The L2 location provides the necessary low gravity-gradient environment for economical formation flying, long observing windows and optimal cooling for the instruments. XEUS can build on the L2 halo experience of Herschel, Gaia and JWST. The present baseline is to fly as a single composite (DSC + MSC) and to separate only after completion of all major orbit manoeuvres (Fig. 5).

\subsection{Spacecraft configuration}

XEUS requires at least a focal length of $\sim 35 \mathrm{~m}$ to reach a collecting area of $5 \mathrm{~m}^{2}$ at $1 \mathrm{keV}$ and a spatial resolution $<5$ arc sec (goal 2 arc sec). A longer focal length would reduce the FoV of the instruments. Given the $35 \mathrm{~m}$ focal length chosen, a dual spacecraft configuration (Fig. 6) is essential. The MSC 
Fig. 4 DSC on top of MSC

inside Ariane medium fairing

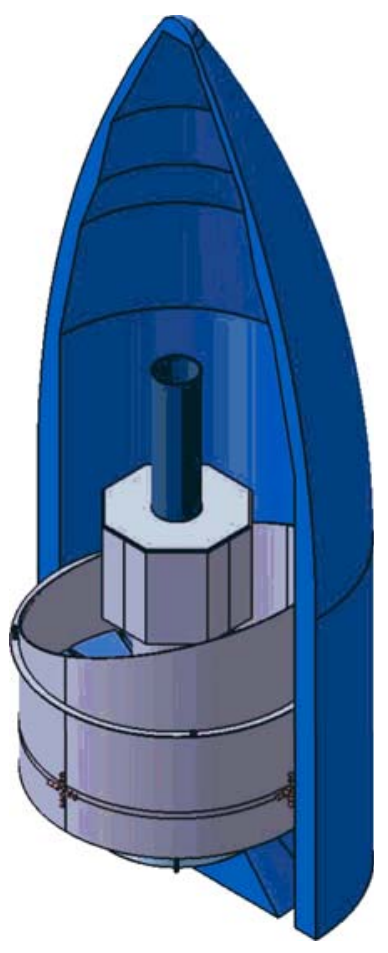

points the optical axis of the mirror at the target, using a classical AOCS with star trackers, etc., and cold gas thrusters (to avoid mirror contamination). The DSC keeps one of the focal plane instruments at the focal point of the mirror. In addition to star trackers, which provide the primary attitude reference, its AOCS uses laser and RF rangefinders, with cold gas thrusters, to maintain the formation flying.

\subsubsection{Mirror spacecraft}

The MSC is three axis stabilised with the XEUS mirror as the sole payload. The mass of the silicon pore optics mirror is $<1,300 \mathrm{~kg}$. The inner $0.67 \mathrm{~m}$ radius of the optics is dictated by the $120 \mathrm{~cm}$ diameter of the Ariane launch adapter. The spacecraft subsystems are contained inside the central thrust cylinder (Figs. 7

Fig. 5 Separation of MSC, DSC, and adapter after arrival at L2

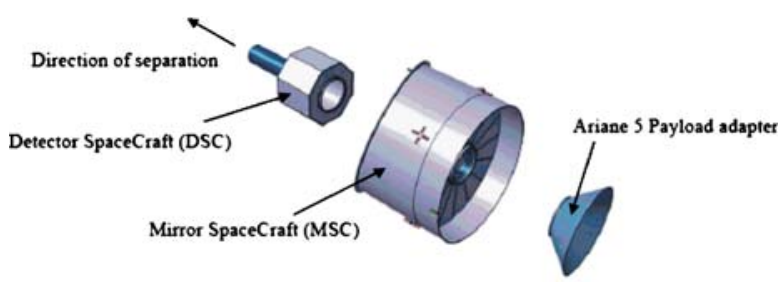


Fig. 6 Artist impression of the mirror spacecraft (right) flying in formation with the detector spacecraft

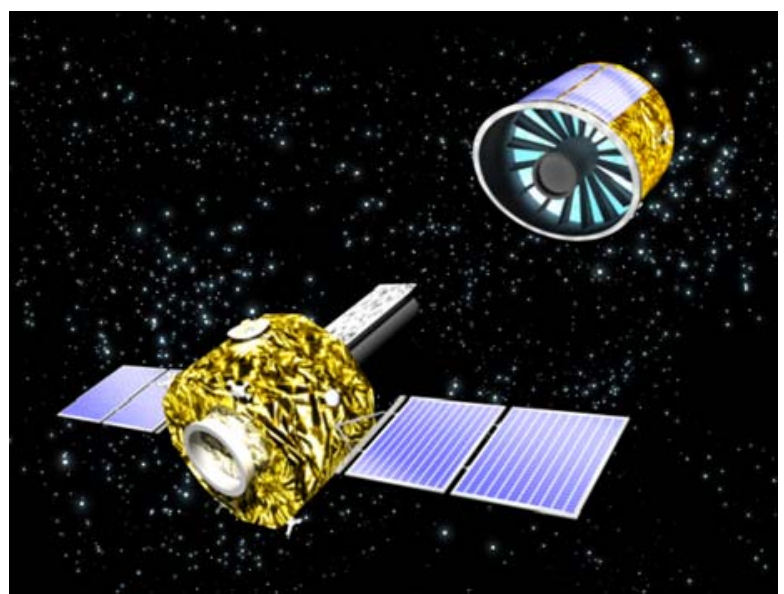

and 8), which attaches to the launcher adapter. The outer radius of $2.1 \mathrm{~m}$ is limited by the diameter of the Ariane fairing. The mirror petals are supported by the radial CRFP structure and the central thrust cylinder. The optics is surrounded by a cylindrical sunshield with solar cells on the sunward half of the sunshield. An annular skirt of $16 \mathrm{~cm}$ is mounted between the mirror and the cylindrical sunshield. This, together with the MSC sunshield and the DSC baffles, blocks X-rays and stray light from the instrument fields of view. The total wet mass of the MSC (including margin) is $4,200 \mathrm{~kg}$.

Fig. 7 On-axis view of the preliminary telescope configuration inside the MSC. The central thrust and the radial vanes support the mirror petals. The mirror petals are surrounded by a cylindrical sun baffle

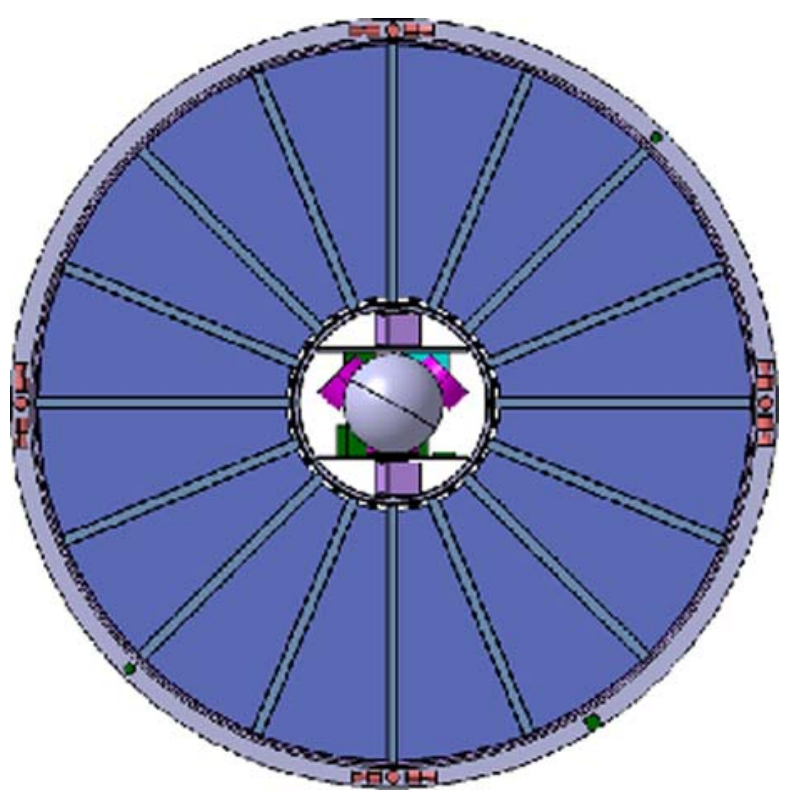


Fig. 8 Exploded view of the preliminary telescope configuration in the MSC, including support structure, mirror petals, contamination covers and $\mathrm{S} / \mathrm{C}$ subsystems. The cylindrical sunshield surrounding the above unit is not shown in this picture

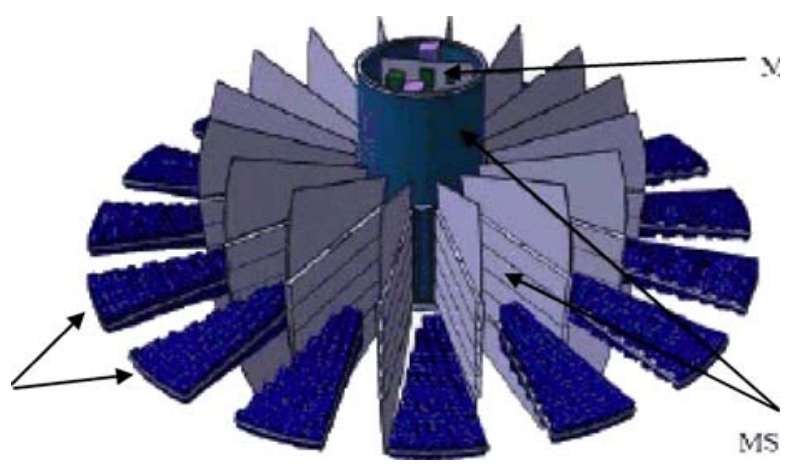

\subsubsection{Detector spacecraft}

The Detector Spacecraft carries the instrumentation payload and the formation-flying package that keeps the prime focus located on the centre of the relevant instrument FoV. The spacecraft is three-axis stabilised and electrical power is provided by deployable solar panels. It also provides the stray light baffles, the cooling necessary for the focal planes, and the main data downlink. Fig. 9 shows a 3D-drawing from one of the industrial accommodation studies of the DSC. Clearly visible is the central baffle of the WFI/HXI that also serves as a structural element for the spacecraft. The main challenges for the DSC are the formation flying package and the cooling system for the NFI. The wet mass of the DSC (including margin) is 2.125 tonnes. The total mass that can be launched by the Ariane $56 \mathrm{ECA}$ is $6,400 \mathrm{~kg}$, which matches the predicted total spacecraft wet mass of $6,325 \mathrm{~kg}$.

Fig. 9 Accommodation of the WFI/HXI with the central $8 \mathrm{~m}$ long baffle. The blue instrument behind the central baffle is the NFI. The lower unit contains the $\mathrm{S} / \mathrm{C}$ subsystems

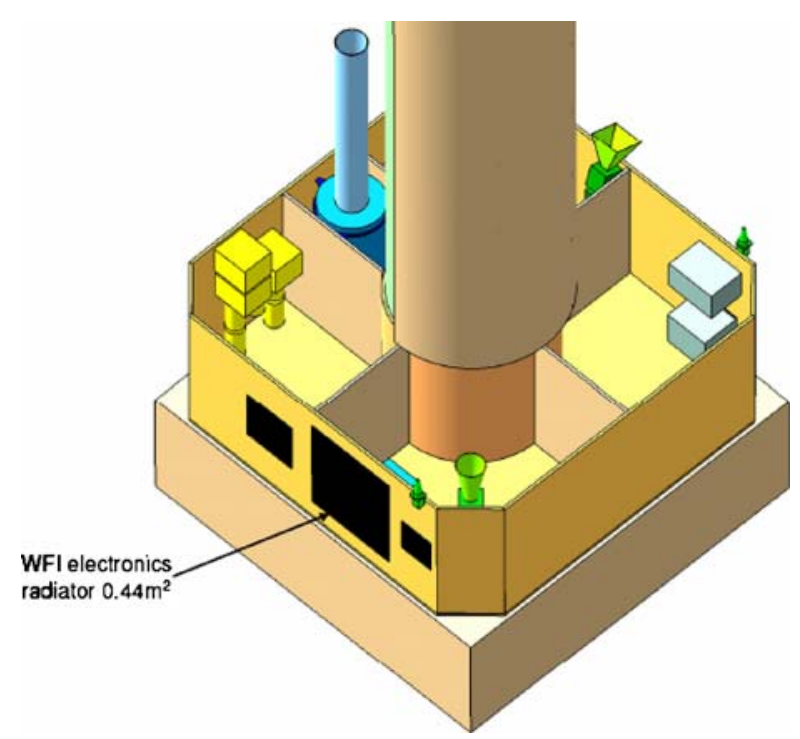




\subsection{Thermal subsystem}

The cooler requirements for the instruments are given in Table 2. Several of the instruments require cooling to $210-260 \mathrm{~K}$. Passive radiative coolers with a Peltier element for temperature control are proposed.

The NFI requires cooling to $50 \mathrm{mK}$ with an effective cooling power between $2-5 \mu \mathrm{W}$. Cryogen-free cooling systems should be used for XEUS given the heritage from Herschel and Planck in Europe, and of Akari, Smiles, and Suzaku in Japan. Given its heritage European industry has come up with two quite different cooling approaches in the XEUS DSC accommodation study. Astrium has proposed a partly redundant system based on two Stirlings from EADS $(300 \longrightarrow 80 \mathrm{~K})$; two Stirlings from EADS $(80 \longrightarrow 18 \mathrm{~K}) ; 2 \mathrm{JT}$ from RAL $(18 \longrightarrow 4 \mathrm{~K})$; dADR from MSSL ( $4 \mathrm{~K} \longrightarrow 50 \mathrm{mK})$. Most of the elements of this chain do actually exist and several have flight heritage. The mass and power allocation is $310 \mathrm{~kg}$ (incl. cryostat) and $700 \mathrm{~W}$. Alcatel has proposed a more progressive approach using passive radiators and $\mathrm{V}$-grooves to get to about $50 \mathrm{~K}$. From there onwards they propose a fully redundant chain based upon two Pulse Tubes from Air Liquide $(50 \longrightarrow 18 \mathrm{~K})$; two He-3 JT from RAL $(18 \longrightarrow 2.5 \mathrm{~K})$; Sorption-cooler based ADR from CEA $(2.5 \mathrm{~K} \longrightarrow 50 \mathrm{mK})$. The He-3 JT does need development. The mass and power allocations for this system are $260 \mathrm{~kg}$ and $470 \mathrm{~W}$.

ISAS/JAXA has longstanding collaboration with Sumitomo for the development and production of cryogen-free coolers for space. A system for XEUS would most likely consist of two two-stage Stirlings $(300 \longrightarrow 18 \mathrm{~K})$; two twostage Stirlings/JT $(18 \longrightarrow 1.8 \mathrm{~K})$; two He-3 sorption coolers $(1.8 \longrightarrow 0.4 \mathrm{~K})$; and one ADR $(0.4 \mathrm{~K} \longrightarrow 50 \mathrm{mK})$. Most of these components do exist and have flight heritage. Mass and Power allocations are about $300 \mathrm{~kg}$ and $450 \mathrm{~W}$. Taking the above numbers on mass and power as a reference, we consider $300 \mathrm{~kg}$ and $600 \mathrm{~W}$ as an adequate resource allocation for the cooling system of the NFI (Table 2). For instrument cooling a Pulse Tube in combination with a double stage Adiabatic Demagnetization Refrigerator has been used, indicating successful shielding for EMI/EMC and sufficient isolation from closed cycle cooler microphonics.

\section{Instrument payload}

\subsection{Model payload}

The science aims of XEUS (Table 1) are very demanding and require state of the art instrumentation. The focal plane plate scale equals $170 \mu \mathrm{m} / \mathrm{arc} \mathrm{sec}$. Furthermore the requirements on spatial resolution, FoV, energy resolution, energy range, quantum efficiency, count rate capability, and polarization sensitivity cannot be met by a single focal plane instrument. Therefore the following instrument complement (Table 2) in the focal plane of the optics, is proposed for the model payload to meet the science objectives: 


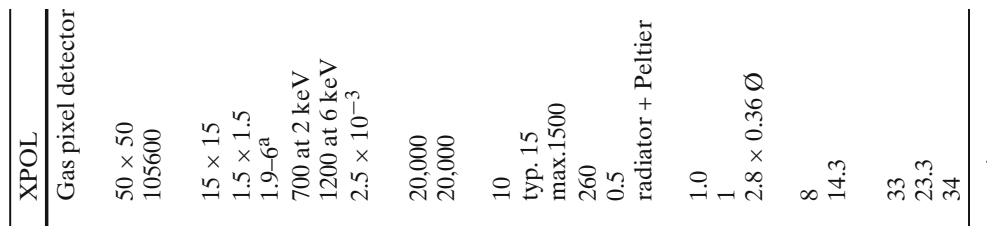

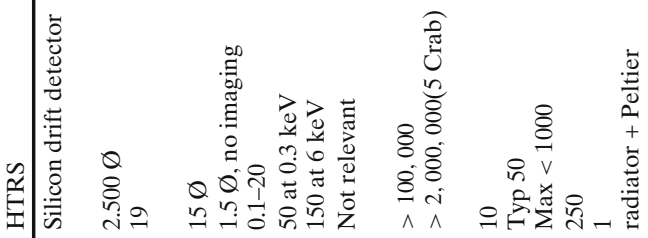

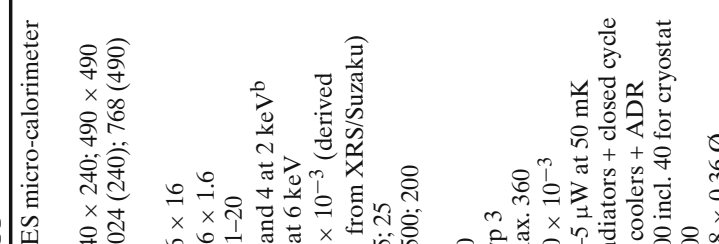

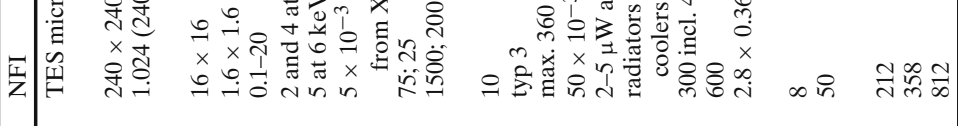

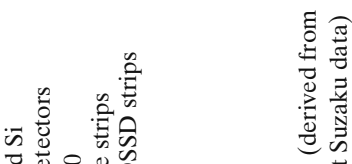

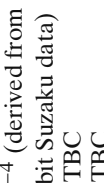

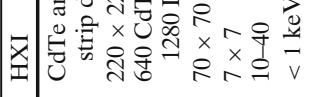

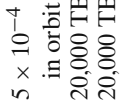

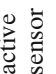

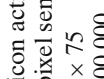

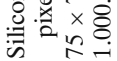

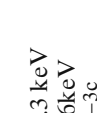

西

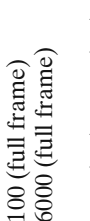

I

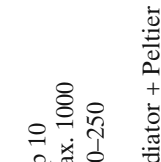

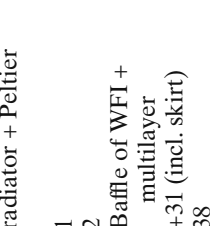

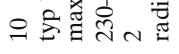

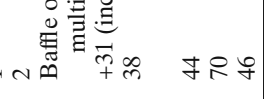


- A WFI covering the $0.05-20 \mathrm{keV}$ energy range with large FoV, excellent spatial resolution and efficiency, good energy resolution, and adequate count rate capability

- A confocal HXI that covers the same FoV with excellent spatial resolution and efficiency in the 10-40 keV energy range, in combination with good energy resolution and count rate performance

- A high spectral resolution imager (NFI) that covers the $0.1-20 \mathrm{keV}$ energy range with unprecedented energy resolution, narrow FoV and relatively low count rate capability

- A non-imaging HTRS that covers the $0.1-20 \mathrm{keV}$ energy range with good energy resolution but ultra-high count rate capability

- An imaging XPOL with a narrow FoV, modest energy resolution, and excellent sensitivity to polarization in the $1.9-6 \mathrm{keV}$ energy range.

\subsection{Sensitivity calculation}

Depending on energy and instrument, the XEUS effective photon collecting area (throughput) is about a factor of 40-50 larger than that of the XMM-Newton low-resolution spectrometric imagers (pn and MOS) and several hundred times larger than that of the XMM-Newton reflection grating (Fig. 13).

The XEUS sensitivity also depends on the background, which consists of unrejected non-X-ray background arising from the radiation environment in which the observatory operates, of the local Galactic "foreground" emission and of the unresolved extragalactic (AGN-related) component. This background scales with the extraction diameter used to search for photons from a point-like source on the sky. For this analysis an $80 \%$ encircled power diameter of $4^{\prime \prime}$ and $10^{\prime \prime} \varnothing$ has been used, depending on the goal ( $2^{\prime \prime}$ half-energy width (HEW)) and requirement (5" HEW) resolution of the telescope, respectively. For the non-X-ray background we conservatively assume $5 \times 10^{-3} \mathrm{cts} \mathrm{cm}^{-2}$ $\mathrm{s}^{-1} \mathrm{keV}^{-1}$ for the WFI, which is the value measured by the XMM pn-CCD camera, and a factor of 10 better for the HXI. The estimate of the local Galactic "foreground" emission is based on the BeppoSAX LEGS results [9]. The unresolved extragalactic (mainly AGN) component is assumed to be $20 \%$ of the hard diffuse X-ray power-law component $10 \mathrm{E}^{-1.42} \mathrm{ph} \mathrm{cm}^{-2} \mathrm{~s}^{-1}$ $\mathrm{keV}^{-1} \mathrm{sr}^{-1}$. This fraction is somewhat arbitrary, as it depends on sensitivity, but it is probably conservative. In particular below $1 \mathrm{keV}$, the excellent energy resolution of the NFI gives a significant advantage, since source emission can be detected in between the "sky lines" of the Galactic foreground.

Combining the telescope throughput with the modelled background rates we can calculate the expected sensitivity for different energy bands as a function of observing time, which is shown in Fig. 10. Depending on the resolution of the telescope, we obviously arrive at different sensitivities. The final sensitivity limit is set by source confusion, which we assume to become important at $\sim 40$ beams per source. Here we define the beam size as the HEW of the point-spread-function (PSF). 
Fig. 10 XEUS WFI and HXI sensitivity as a function of observing time for different energy bands. The requirement $\left(5^{\prime \prime}\right.$, dashed $)$ and goal ( $2^{\prime \prime}$, solid $)$ angular resolution have been assumed for all cases. For the lower angular resolution cases, the sensitivity degrades due to the higher background and ultimately in the $0.5-2 \mathrm{keV}$ band due to the source confusion limit

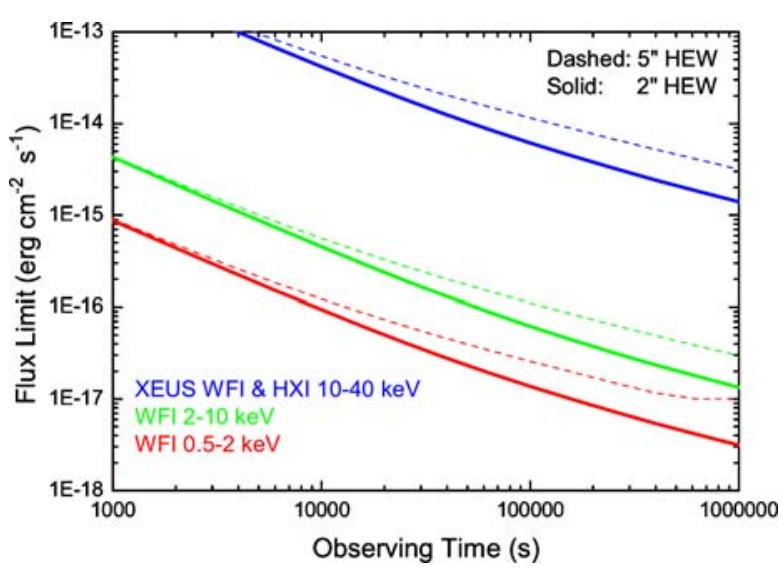

\subsection{Key characteristics of instruments}

\subsubsection{X-ray optics}

The XEUS mirror is the heart of the XEUS mission and its challenging requirements (Table 3) call for the development of new technology. NASA's Chandra Observatory has an angular resolution of 0.5 arc sec with a collecting area of only $\sim 400 \mathrm{~cm}^{2}$ at $1 \mathrm{keV}$ and an area-to-mass ratio of $\sim 0.8 \mathrm{~cm}^{2} / \mathrm{kg}$. ESA's XMM-Newton Observatory consists of three identical mirror systems with each $\sim 1400 \mathrm{~cm}^{2}$ at $1 \mathrm{keV}$ with a modest angular resolution of $\sim 15 \operatorname{arc~sec}$ and an area-to-mass ratio of $\sim 6 \mathrm{~cm}^{2} / \mathrm{kg}$. The huge area required for XEUS and the limits set on mass by affordable launchers require development of optics with an area-to-mass ratio of about $35 \mathrm{~cm}^{2} / \mathrm{kg}$. So the challenge is to produce a mirror system with a significantly larger area to mass ratio than provided by current technology, while at the same time retaining a high angular resolution. The idea of using square pore optics in an approximate form of Wolter Type I geometry was suggested in 1998 [10], but at the time such optics could only be manufactured from glass which provides a very favourable area to mass ratio but was, and still is, unable to meet the high angular resolution requirements of XEUS. While progresses are expected from ongoing glass technology developments, the use of Silicon wafers from semiconductor industry [11] currently appears as the most promising solution.

Table 3 XEUS X-ray optics requirements

\begin{tabular}{lll}
\hline Item & Requirement & Goal \\
\hline Angular resolution HEW & 5 arc sec & 2 arc sec \\
Collecting area at $1 \mathrm{keV}$ & $5 \mathrm{~m}^{2}$ & $5 \mathrm{~m}^{2}$ \\
Collecting area at $7 \mathrm{keV}$ & $2 \mathrm{~m}^{2}$ & $2 \mathrm{~m}^{2}$ \\
Collecting area at $30 \mathrm{keV}$ & $400 \mathrm{~cm}^{2}$ & $1000 \mathrm{~cm}^{2}$ \\
Field of view diameter & $20(1 \mathrm{keV}) ; 15(7 \mathrm{keV}) ; 10(30 \mathrm{keV})$ \\
\hline
\end{tabular}


Silicon pore optics can provide the lightweight high-resolution X-ray mirrors for XEUS. The pore optics is manufactured using highly polished $(\sim 3 \AA$ rms roughness) and flat $\left(<0.2 \mu \mathrm{m}\right.$ over $\left.25 \times 25 \mathrm{~mm}^{2}\right)$ silicon wafers of $750 \mu \mathrm{m}$ uniform thickness ( $<3 \mu \mathrm{m}$ PTV) and $30 \mathrm{~cm}$ diameter. These wafers are produced as standard items by industry and most fortuitously have a surface finish and figure which are tailor-made for X-ray optics. The wafers are cut into rectangular sheets $\sim 10 \times 10 \mathrm{~cm}^{2}$ and ground and polished on one face to introduce a small taper in thickness $(\sim 1 \mu \mathrm{m}$ change in thickness from edge to edge). Rectangular channels or grooves (which will become the pores) are cut using a computer controlled diamond saw across one face of each wafer. The uprights between the grooves will form the sidewalls of the pores. These surfaces and the bottom of the grooves are left rough by the sawing process, which neatly suppresses unwanted X-ray reflections. The opposite surface of each wafer, without the grooves, is then coated with high- $\mathrm{Z}$ material ( $\mathrm{Ir}, \mathrm{Pt}, \mathrm{Au}$ or a multi-layer structure) and possibly overcoated with a thin layer of carbon. The coated surfaces will provide the X-ray mirror reflecting surface within each rectangular pore.

The wafers are then robotically assembled into a stack using a conically profiled mandrel to set the accurate form required for the approximate Wolter I geometry. As each wafer is bent and introduced into the stack it is bonded to the previous wafer by a cold-weld process. This is initially an optical bond because the surfaces (the top of the sidewalls on one wafer and the bare strips between the coating on the opposite wafer) are extremely flat and clean. Additional application of pressure and/or heat produces a partial chemical bonding. Up to 100 wafers are integrated to form a complete high performance pore optic (HPO). Many further details of the processes required to produce

Fig. 11 Prototype XOU X-ray lens-courtesy Cosine Research

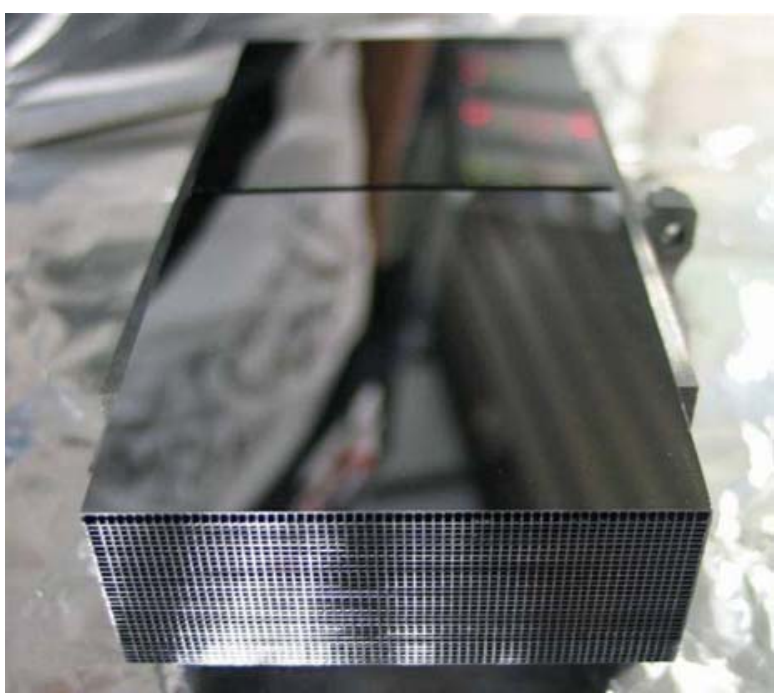


the HPOs are given in [12]. The programmatic required for the large-scale production of the silicon pore optics is discussed in [13].

Two HPOs must be assembled and co-aligned in a tandem pair or X-ray Optical Unit (XOU) (Fig. 11). X-rays are reflected once from the tangential outer walls of the rectangular pores in the first HPO and once from the corresponding surfaces in the second HPO. The HPOs are aligned to form a conical approximation to the Wolter I grazing incidence geometry. Each XOU therefore acts as an off-axis X-ray lens with aperture dimensions $\sim 10 \times 10 \mathrm{~cm}^{2}$ and $\sim 20 \mathrm{~cm}$ axial length. Each XOU must be positioned and aligned in modules (petals), which together form an optic with a single X-ray focus. It is remarkable that once the reflecting surfaces within an XOU are correctly aligned, the alignment of individual XOUs in the petal structure is much less critical. The assembly of silicon pore optics into a modular structure is discussed by [14].

The size of the pores, the size of the annular aperture, and the focal length determine the collecting area as a function of energy and the limiting angular resolution that can be achieved. The effective collecting area of each XOU depends on the size of each pore, the thickness of the pore walls, the grazing angles of reflection, the surface coating, and the X-ray energy. A substrate and rib thickness of $150 \mu \mathrm{m}$ and wafer thickness of $750 \mu \mathrm{m}$ enables a pore structure of $0.6 \times 1.5 \mathrm{~mm}$ with $\sim 70 \%$ aperture efficiency, which results in a low energy collecting area of $\sim 70 \mathrm{~cm}^{2}$ for an XOU. An overcoat of carbon on the iridium-coated reflecting surfaces of HPOs can provide enhanced low energy reflectivity [15].

To obtain a low energy collecting area of $\sim 5 \mathrm{~m}^{2}$, about 1,000 XOUs must be assembled into a dense array of X-ray lenses covering an annular aperture. An inner annular radius of $0.67 \mathrm{~m}$, limited by the MSC launch adapter, accommodates HPOs with small grazing angles such that the collecting area at $10 \mathrm{keV}$ is $\sim 0.8 \mathrm{~m}^{2}$. If multi-layer coatings are used on the inner HPO the high energy response can be extended to give $\sim 0.1 \mathrm{~m}^{2}$ at $30 \mathrm{keV}$ [15]. An outer aperture radius of $2.1 \mathrm{~m}$ can be accommodated within the launcher fairing including a baffle skirt of width $\sim 16 \mathrm{~cm}$.

For such a configuration a minimum focal length of $\sim 35 \mathrm{~m}$ is required to give an effective area of $2 \mathrm{~m}^{2}$ at $7 \mathrm{keV}$. Larger focal length increases the effective area at the higher energies, but also the mass of the optics. The chosen pore size is consistent with a diffraction limited angular resolution $<2$ arc sec (see Table 4 for a summary on optics characteristics).

Table 4 Optics characteristics

\begin{tabular}{ll}
\hline Characteristic & Value \\
\hline Pore size & $0.6 \times 1.5 \mathrm{~mm}^{2}$ \\
Aperture radii & $0.67-2.1 \mathrm{~m}$ \\
Grazing reflection angles & $0.27-0.86^{\circ}$ \\
Focal length & $35 \mathrm{~m}$ \\
Plate scale & $170 \mu \mathrm{m} / \mathrm{arc} \mathrm{sec}$ \\
\hline
\end{tabular}


Table 5 Optics error budget

\begin{tabular}{llllll}
\hline Specification $(\operatorname{arc~sec})$ & Inherent & Intrinsic & Extrinsic & Environment & Total \\
\hline Goal & 1.4 & 1.2 & 0.5 & 0.5 & 2.0 \\
Requirement & 1.8 & 3.7 & 2.0 & 2.0 & 5.0 \\
\hline
\end{tabular}

Contributions to the operational angular resolution can be sub-divided into 4 categories: (1) the inherent properties of the conical approximation to the Wolter I geometry and diffraction as discussed in [16, 17], (2) intrinsic manufactured properties of the individual HPOs/XOUs or X-ray lenses, (3) extrinsic elements involving the alignment of the XOUs into the full mirror assembly and (4) environmental effects including thermal distortions, alignment of the mirror and detector spacecraft, stability of the aspect solution etc.. First-cut estimates of the HEW contributions in arc sec are given in Table 5. The major factors which must be included in the angular resolution budget are intrinsic (e.g. conical approximation, figure errors, micro-roughness and alignment errors, in particular for the two HPOs), extrinsic (e.g. the alignment of XOUs into the aperture array), and environmental (e.g. thermal loading, spacecraft positioning errors and boresighting). The inherent and intrinsic terms associated with the design and manufacturing of the XOUs are dominant. In order to reach the goal of 2 arc sec it may be necessary

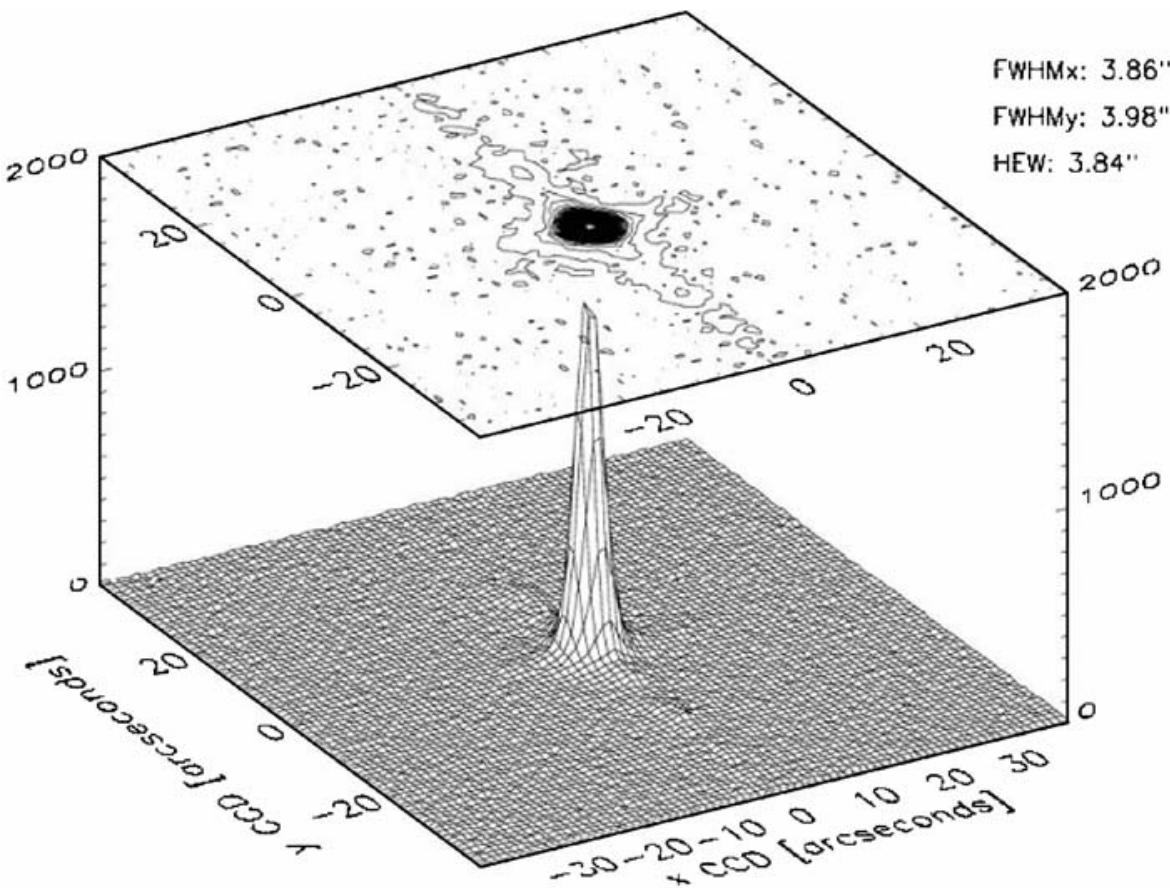

Fig. 12 The PSF of the first prototype XOU derived by pencil beam testing at BESSY. The effective HEW is 3.84 arc sec but after allowing for the figure errors known to exist in the mandrel used for the HPO stacking the effective HEW is 2.6 arc sec 
to reduce the effect of the conical approximation using smaller pores and thereby reducing the length of the reflecting surfaces: this is part of the optimization task.

Figure 12 shows the synthesized PSF for the first prototype XOU as derived from pencil beam measurements. A local effective HEW of 3.8 arc sec in this first try is very encouraging when compared with the requirement of $3.7 \mathrm{arc} \mathrm{sec}$. The result displayed is not definitive because the prototype does not include high- $\mathrm{Z}$ coating or tapering to give a conical approximation to a Wolter Type I geometry and there are aspects of the geometry which are not probed by this test. However, the signs are very encouraging and indicative that the intrinsic error allocation can be met. Further development and testing is required to fully characterise the limiting resolution performance of the XOUs.

The total mirror mass is dominated by the Si plates and is critically dependent on the size of the pores and the thickness of the walls. We have estimated the mass required to meet the effective area requirements at 1 and $7 \mathrm{keV}$ using the current parameters for the Si pore manufacture and assuming an aperture annulus of $0.67 \mathrm{~m}<R<2.10 \mathrm{~m}$ with an effective dead area based on existing petal blocking fractions. We will require $\sim 30,000 \mathrm{Si}$ wafers cut into $\sim 200,000 \mathrm{Si}$ plates and integrated into $\sim 1000$ XOUs. In addition we include the structure mass required to support the $\mathrm{Si}$ and mass required for ancillary items like X-ray/optical baffles, a Sun-shield, thermal control items integrated into the mirror assembly and possibly a background particle rejection system (Table 6).

The predicted on-axis collecting area as a function of energy, using an iridium reflection surface with a carbon over-coat on the $\mathrm{Si}$ units used to derive the mass estimates, is shown in Fig. 13. The area to mass ratio at $1 \mathrm{keV}$ provided by the Si pore design is $\sim 38 \mathrm{~cm}^{2} / \mathrm{kg}$ which is the critical advantage for this technology. The carbon over-coat fills in the dips due to the Iridium absorption edges at $\sim 2 \mathrm{keV}$ and enhances the low energy effective area. If the reflecting surfaces of the XOUs near the inner edge of the aperture are coated with the appropriate multlilayers, $\mathrm{Pt} / \mathrm{C}$ for example, the energy response can be extended to $\sim 30 \mathrm{keV}$ as indicated. Optimization of the multilayer design and using a smaller inner radius for the aperture (fixed at $0.67 \mathrm{~m}$ in the present design) may help to achieve the goal of $\sim 1,000 \mathrm{~cm}^{2}$ at $30 \mathrm{keV}$.

\subsubsection{The WFI}

As the main focal plane imaging detector, the purpose of the WFI is to provide $7 \times 7$ arc $\min ^{2}$ images in the $0.1-20 \mathrm{keV}$ energy band with spectral and time resolved photon counting. Since the plate scale for $35 \mathrm{~m}$ focal length equals $170 \mu \mathrm{m} / \mathrm{arcsec}$, the chosen pixel size of $75 \mu \mathrm{m}$ is enough to oversample images

Table 6 Optics mass budget

\begin{tabular}{llll}
\hline Si & Support & Ancillary & Total \\
\hline $882 \mathrm{~kg}$ & $176 \mathrm{~kg}$ & $238 \mathrm{~kg}$ & $1296 \mathrm{~kg}$ \\
\hline
\end{tabular}




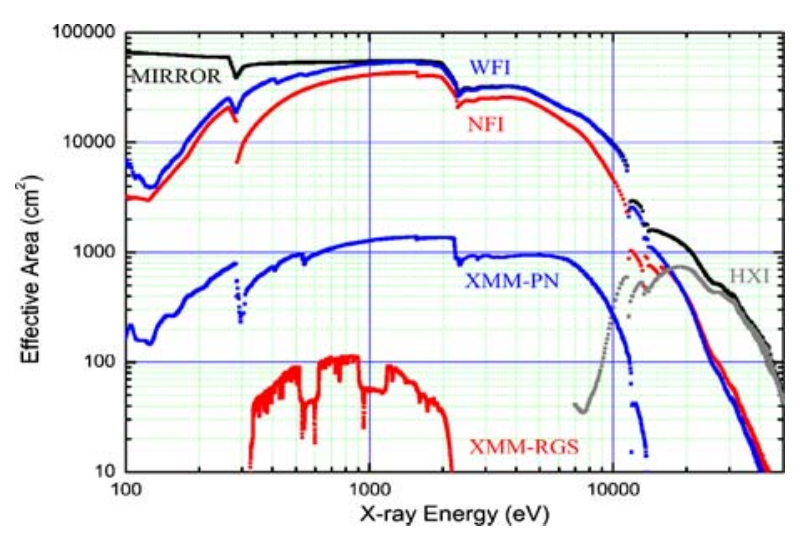

Fig. 13 Effective area of the X-ray optics alone and the X-ray optics in combination with the various instruments. The area of the WFI and HTRS are actually equal and are $>5 \mathrm{~m}^{2}$ between 1-2 $\mathrm{keV}$. The area of the NFI peaks at about $4 \mathrm{~m}^{2}$ between 1-2 keV. All instruments have lower collecting area below $1 \mathrm{keV}$, because of the necessary entrance windows and filters. At energies up to about $15 \mathrm{keV}$ the WFI, NFI, and HTRS closely follow the area provided by the optics. Above about $15 \mathrm{keV}$ the HXI takes over. The areas for the XMM-pn and for both XMM-RGSs are shown for comparison. Note the factor of 30-40 between XMM-PN and XEUS-WFI, and the factor of 400 between the XMM-RGSs and the XEUS-NFI

of 2 arc sec HEW resolution. Design drivers for the wide field imager are the large number of pixels combining a wide FoV with high spatial resolution, in combination with high read-out speed, good energy resolution and high quantum efficiency over a large energy range. Homogeneous response to radiation and unprecedented long term stability are also essential. This required the development of new Active Pixel Sensor technology, allowing for the read-out of partial and full images simultaneously. The WFI proposed is an active pixel sensor based on the Depleted p-channel Field Effect Transistor (DEPFET) concept [18]. As charge transfer in the detector body is not needed due to the local amplification of the signals, Charge Transfer Inefficiency related radiation damage is no longer an issue. The detection sensitivity is determined by the large effective area, the high spatial resolution, and the low background of the instrument. Given the two-spacecraft configuration for XEUS, X-rays originating from the sky area outside the telescope FoV could contribute to the instrument background. To restrict this, the instrument requires a $7.9 \mathrm{~m}$ long baffle of $1.0 \mathrm{~m}$ diameter in combination with a $16 \mathrm{~cm}$ skirt mounted on the MSC. About $107 \mathrm{~kg}$ is estimated using a crude design with the baffle being a structural element of the DSC. A detailed baffle design activity, optimizing MSC skirt width versus baffle length could further reduce baffle length and mass or result in a potential increase in the WFI FoV.

The WFI meets the scientific requirements as given in Table 1 . The sensitivity of $3 \times 10^{-18} \mathrm{erg} \mathrm{s}^{-1} \mathrm{~cm}^{-2}$ can be achieved by the current design within a 1 Ms observation time (Fig. 10) and 2 arc sec spatial resolution. The effective area is shown in Fig. 13. 
Fig. 14 Energy response at 5.9 and $6.4 \mathrm{keV}$ from $55 \mathrm{Fe}$ X-rays

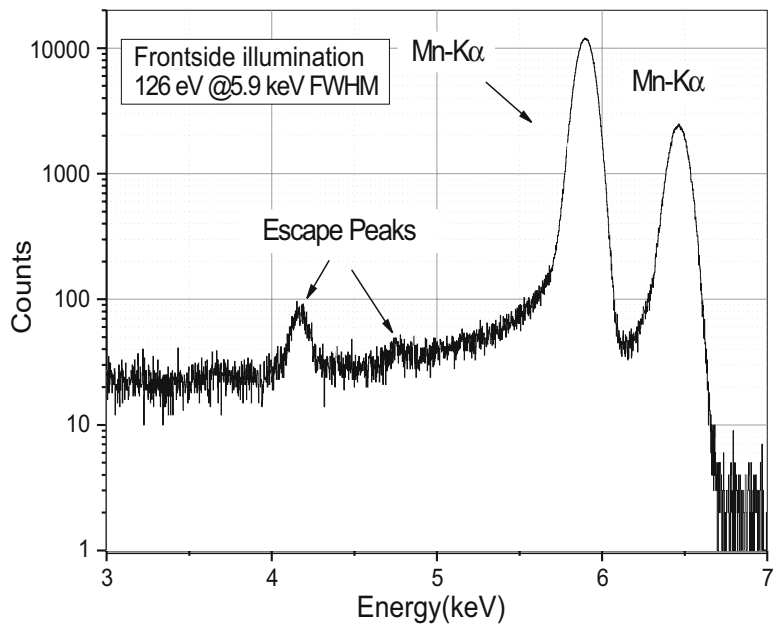

The DEPFET (see Figs. 14 and 15), the core of the WFI instrument is being developed by MPE Garching not only for XEUS but also for other $\mathrm{X}$-ray instruments/missions carried out in collaboration with the University of Leicester (BepiColombo) and the University of Tübingen (Simbol-X). The MPI-HLL, jointly operated by MPE and the MPI for physics, is already working on the integral camera design of all three missions. The high speed ASICs are currently developed in cooperation with the electronics groups of Politecnico di Milano/INFN. International partners with expertise in the field will be invited to join the WFI development.

\subsubsection{The HXI}

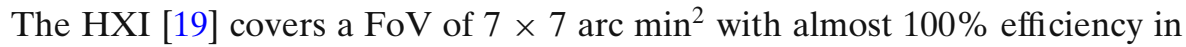
the $10-40 \mathrm{keV}$ band with $\Delta E<1 \mathrm{keV}$ up to $40 \mathrm{keV}$ (Fig. 16). To meet this

Fig. 15 Breadboard showing a DEPFET array with clocking and read-out electronics

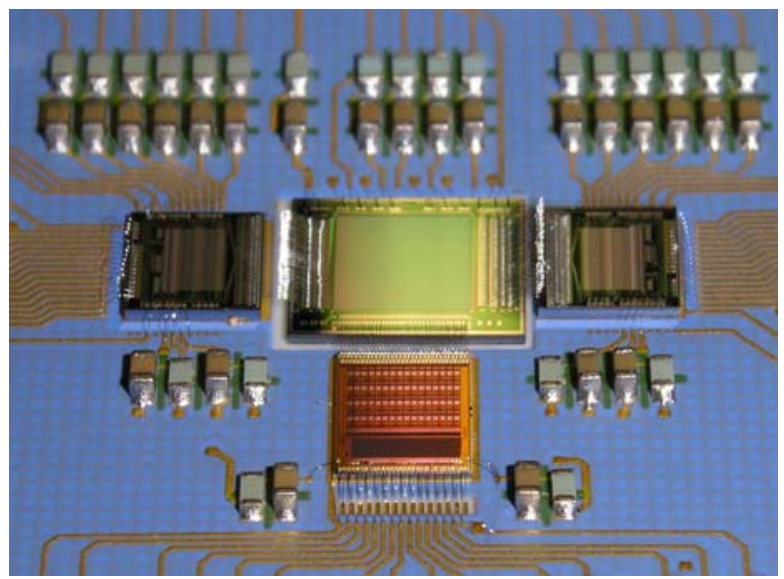


Fig. 16 Energy spectrum for CdTe pixel detector read-out with custom design ASIC

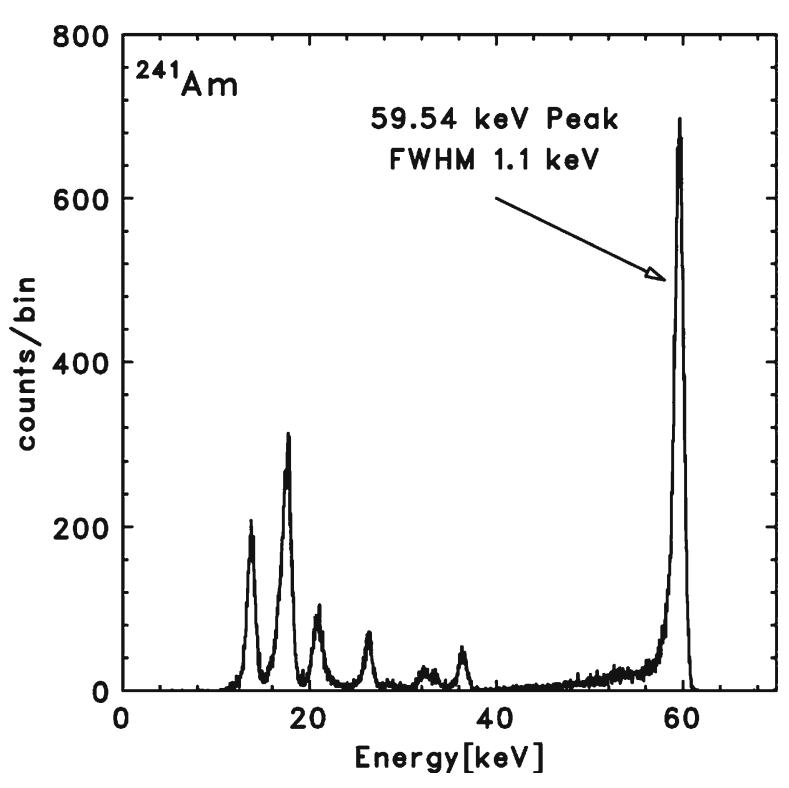

quantum efficiency, $0.5 \mathrm{~mm}$ thick double-sided CdTe strip detectors are proposed. The $70 \times 70 \mathrm{~mm}^{2}$ large FoV detector is built up from $200 \mu \mathrm{m}$ wide strips to guarantee excellent imaging. To observe the $5-40 \mathrm{keV}$ energy band simultaneously, the HXI is directly mounted below the WFI (Fig. 17). In order to obtain high sensitivity (low background), the CdTe detector is actively shielded by a $2 \mathrm{~cm}$ thick BGO scintillator. A full background rejection system, composed of active and passive graded shield is also in study at CEA and APC, Paris, taking into account the mission design of Simbol-X, Monte-Carlo simulations, and measurements from the Integral/IBIS mission. At least one layer of a Double-sided Silicon Strip Detector (DSSD) is planned between the CdTe detector and the WFI. This shields the WFI from fluorescence lines

Fig. 17 Schematic drawing of the integration of HXI behind WFI

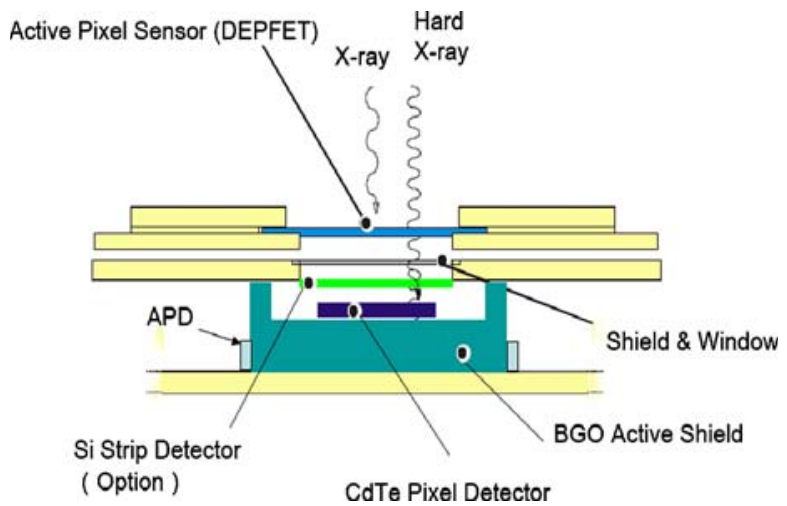

Springer 
emitted by $\mathrm{CdTe}$, and acts as an excellent imaging spectrometer for photons around $10-20 \mathrm{keV}$.

Both the CdTe and the DSSD are read-out by the same ASICs that requires only $250 \mu \mathrm{W} /$ channel. The collimator (baffle and skirt) for the WFI has to be made opaque to X-rays up to $40 \mathrm{keV}$. A high-Z tapered metal coating of the WFI baffle with total weight of about $31 \mathrm{~kg}$ will be required. The HXI meets the scientific requirements for the mission as formulated in Table 1. The effective area is shown in Fig. 13. In the case of the HXI, the European PI of the WFI will receive the HXI from Japan and integrate it with the WFI before delivery to the prime contractor.

ISAS/JAXA has been developing the HXI, and a breadboard with excellent performance exists. Since the concept of the HXI on XEUS comes from the HXI on the Japanese NeXT mission, which is proposed to be launched in 2012, most of the detector components can be prepared based on the experiences with NeXT. International partners with expertise in the field will be invited to join the HXI developments.

\subsubsection{The NFI}

This instrument offers imaging spectroscopy with excellent energy resolution and high detection efficiency in the $0.1-20 \mathrm{keV}$ band over a $1.6 \times 1.6$ arc $\min ^{2} \mathrm{FoV}$. The instrument proposed is based on micro-calorimeter pixel arrays with normal-to-superconducting phase transition thermometers, often called transition-edge-sensors (TES) [20]. Recent experiments at the BESSY synchrotron facility show that the TES technology enables excellent performance over the complete XEUS energy band (Figs. 18 and 19). Therefore the need for a second NFI, based upon superconducting tunnelling junction (STJ) detectors covering the low energy range is no longer a pressing issue.

To cover a $1.6 \times 1.6 \operatorname{arc~min}^{2} \mathrm{FoV}$, a central imaging array of $32 \times 32$ pixels of $240 \mu \mathrm{m}$ size $\left(0.8 \times 0.8 \mathrm{arc} \mathrm{min}^{2}\right)$ and $100 \mu$ s fall time is surrounded by

Fig. 18 Single pixel response to $0.25 \mathrm{keV} \mathrm{X}$-rays with 1.58 eV FWHM energy resolution. Low energy tail is due to X-ray absorption outside the TES-absorber, which will be solved for new array layouts

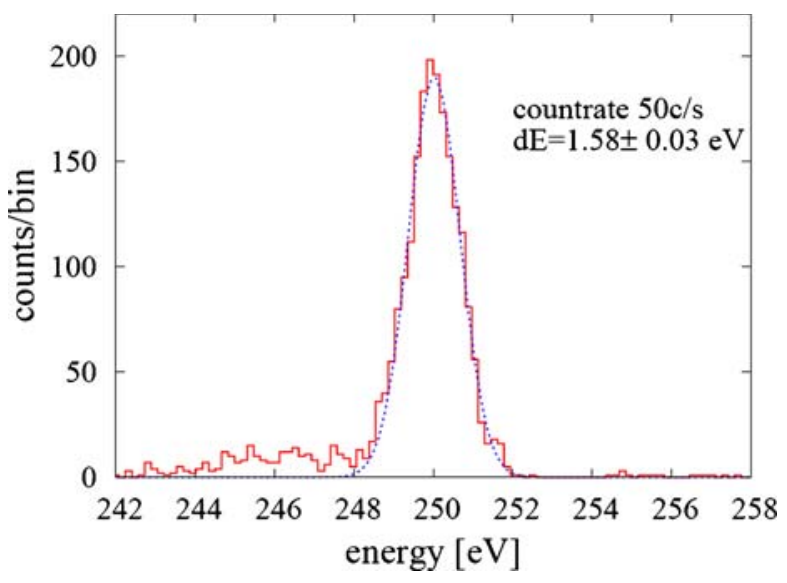


Fig. 19 Single pixel response to $\mathrm{Mn}-\mathrm{K} \alpha_{1,2} \mathrm{X}$-rays at $5.9 \mathrm{keV}$ with $2.52 \mathrm{eV}$ FWHM energy resolution. Low energy tail is due to absorption outside pixel absorber

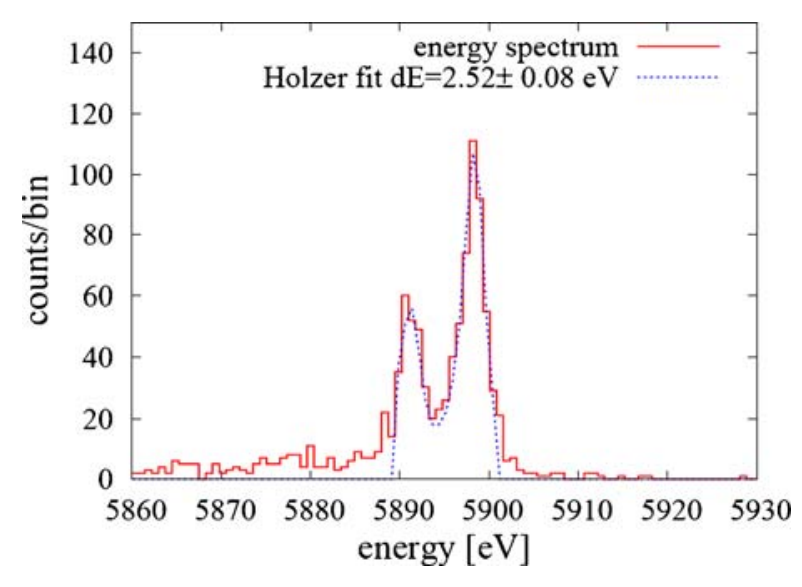

768 pixels of $490 \mu \mathrm{m}$ size and $300 \mu$ s fall time. An anti-coincidence detector is mounted behind the pixel array. The non-rejected background will be dominated by secondary events produced in the spacecraft, and cryostat. A careful design (material choice, graded shields) in combination with Geant simulations and follow-on tests should result in a background level similar to Suzaku XRS, i.e. $5 \times 10^{-3}$ counts $\mathrm{cm}^{-2} \mathrm{~s}^{-1} \mathrm{keV}^{-1}$, although the $\mathrm{L} 2$ orbit has a $2-3 \times$ higher background than the near earth orbit of Suzaku ( $\sim 0.9$ counts $\mathrm{cm}^{-2} \mathrm{~s}^{-1}$ ). The instrument requires cooling to $50 \mathrm{mK}$ for the sensor array with the cold electronics, and anchor harness requiring mounting at 1 and $4 \mathrm{~K}$, respectively. Instrument heat loads are about $2 \mu \mathrm{W}$ at $50 \mathrm{mK}$ and $1 \mathrm{~mW}$ at $4 \mathrm{~K}$. The 1792 pixels will be read-out by about 44 electronics channels making use of frequency-domain-multiplexing (FDM) in the 1-10 MHz band. Base-band feedback will be used to linearize the intrinsically nonlinear SQUID-response. This approach significantly reduces the required harness and consequently the thermal load on the cooling system. To achieve the required sensitivity the improvement in background rejection efficiency $(2-3 \times)$ above what was achieved on Suzaku will be crucial.

For the NFI SRON is developing a $5 \times 5$ pixel array prototype instrument (EURECA) with several international partners. Presently EURECA contributions come from INA/Zaragoza (LC-filter tuning), ICMA/Zaragoza and IMM/Madrid (MoAu-bilayers), VTT/Helsinki (SQUIDs/FEE), PTB/Berlin (SQUIDs), TMU/ISAS/Tokyo (Electronics), INFN/Genova (TES/electronics), INAF/Rome and INAF/Palermo (calibration), IFCA/Santander and Geneva Observatory/ISDC (Software). The consortium is open to any appropriate international collaboration.

The overall performance demonstration of a $5 \times 5$ array read-out with FDM is planned for end 2008 (EURECA Program). Additional effort will be devoted to LC-filters, SQUIDs, FDM with base-band feedback, and cold head design in a TRP and a GSTP contract. Parallel studies are also devoted to STJ and MIS (CEA/Saclay/Grenoble) technology. 


\subsubsection{The HTRS}

This instrument will provide time resolved single photon spectroscopy for bright point sources up to count rates of $2 \mathrm{Mc} \mathrm{s}^{-1}$. It is based on a Silicon Drift Detector (SDD) [21]. The SDD is a high speed non-imaging spectrometer enabling count rates of up to $10^{5} \mathrm{c} \mathrm{s}^{-1}$ pixel $^{-1}$ with good energy resolution close to Fano limited performance for silicon. A 19-pixel SDD is proposed to meet the requirements. The instrument can have a particularly high bit rate of $500 \mathrm{kbits} \mathrm{s}^{-1}$, which on special occasions should be telemetered directly. In normal operation the data will stream in a 19 Gbit memory and will be sent to the ground at about $50 \mathrm{kbit} \mathrm{s}^{-1}$. The instrument must be operated out of focus by about $10 \mathrm{~cm}$ to distribute the X-ray flux over the full detector array (1.5 cm diameter). CESR is expected to build the HTRS (focusing on the FEE and DPU), with the detector provided by the MPE/MPI-HLL. International partners with expertise in the field will be invited to join the HTRS consortium. There are no critical items for this instrument.

\subsubsection{The XPOL}

The X-ray Polarimeter is based on a gas pixel detector with $50 \mu \mathrm{m}$ pixel size $[22,23]$. The $\mathrm{X}$-ray photon is absorbed by an atom of the gas and a photoelectron is ejected in a direction which carries the memory of the polarization direction of the photon. The secondary electrons of the ionization track created in the gas by the photoelectron are drifted to a Gas Electron Multiplier, which amplifies the charges in a proportional manner. These are then collected by an array of metal pads, the pixels of this imaging device. These pads are the top layer of an ASIC VLSI chip, including a complete

Fig. 20 Measured event track with interaction point and emission direction reconstruction

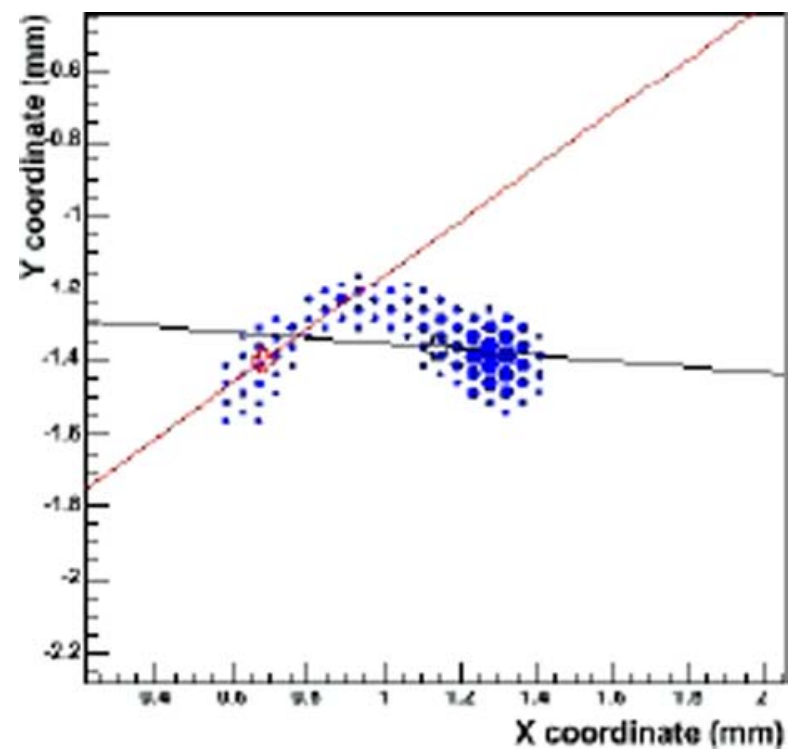


Fig. 21 Measured modulation curve for nearly $100 \%$ polarized photons at $5.4 \mathrm{keV}$ in He-DME (20-80) mixture

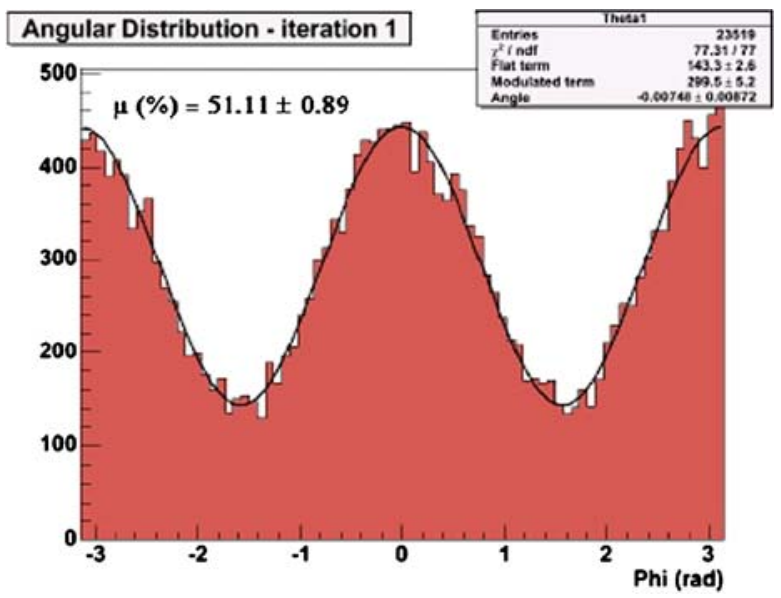

read-out chain for each pad, amplifying the charge collected. An algorithm identifies the interaction point and evaluates the emission direction of the track. The performance of the detector as a polarimeter is based mainly on the quantum-efficiency and on the modulation factor. The selected thickness and composition of the gas-mixture is a trade-off to maximize the sensitivity for a source with a photon spectrum proportional to $\mathrm{E}^{-2}$ (Figs. 20 and 21).

The measured performance already meets the scientific objectives. More gas mixtures will be simulated and a subset tested to try to extend the energy band to lower energies to increase the sensitivity for soft sources (isolated neutron stars, blazars) and to higher energies, mainly to improve energy resolved polarimetry. The detector and interface electronics will be built by INFN/Pisa. A potential contractor for the gas cell manufacture is Oxford Instruments, FI, while the Control Electronics can be outsourced to Alcatel Alenia Space; Italy. International partners with expertise in the field will be invited to join the XPOL consortium. There are no critical items.

Acknowledgements The authors wish to express their appreciation to all those scientists who supported XEUS throughout the years and contributed with suggestions and constructive criticism to the writing of this final proposal. Each author acknowledges partial financial support from their national institutions and agencies from the birth of the original idea to the completion of the proposal, and A. Parmar for acting as a very helpful secretary of the XEUS Science Advisory Group. G.G.C. P. gratefully acknowledges the enthusiastic support from ESA International Space Station department during the early phases of the XEUS concept growth. Without their help XEUS probably would have never made it to successfully respond to the Cosmic Vision call.

\section{References}

1. Worsley, M.A., et al.: The unresolved hard X-ray background: the missing source population implied by the Chandra and XMM-Newton deep fields. MNRAS 357, 1281 (2005)

2. Gilli, R., Comastri, A., Hasinger, G.: The synthesis of the cosmic X-ray background in the Chandra and XMM-Newton era. A\&A 463, 79 (2007) 
3. Di Matteo, T., Springel, V., Hernquist, L.: Energy input from quasars regulates the growth and activity of black holes and their host galaxies. Nature 433, 604 (2005)

4. Cen, R., Ostriker, J.P.: Where are the baryons? II. Feedback effects. ApJ 650, 560 (2006)

5. Nicastro, F., et al.: The mass of the missing baryons in the X-ray forest of the warm-hot intergalactic medium. Nature 433, 495 (2005)

6. Rasmussen, A.P.: On the putative detection of $\mathrm{z}>0$ X-ray absorption features in the spectrum of Mrk 421. ApJ 656, 129 (2007)

7. McNamara, B.R., Nulsen, P.E.J.: Heating hot atmospheres with active galactic nuclei. ARA\&A 45, 117 (2007)

8. Armitage, P.J., Reynolds, C.S.: The variability of accretion on to Schwarzschild black holes from turbulent magnetized discs. MNRAS 341, 1041 (2003)

9. Parmar, A.N., et al.: The low-energy cosmic X-ray background spectrum observed by the BeppoSAX LECS. A\&A 345, 611 (1999)

10. Willingale, R., et al.: Hard X-ray imaging with microchannel plate optics. Exp. Astron. 8, 281 (1998)

11. Beijersbergen, M., et al.: Silicon pore optics: novel lightweight high-resolution X-ray optics developed for XEUS. Proc. SPIE 5488, 868 (2004)

12. Günther, R., et al.: Production of silicon pore optics. Proc. SPIE 6266, 626619 (2006)

13. Kraft, S., et al.: Programmatics of large scale production of silicon pore optics for future X-ray telescopes. Proc SPIE 6266, 626617 (2006)

14. Graue, R., et al.: Assembling silicon pore optics into a modular structure. Proc. SPIE 6266, 61661U (2006)

15. Pareschi, G., et al.: Astronomical soft X-ray mirrors reflectivity enhancement by multilayer coatings with carbon overcoating. Proc. SPIE 5488, 481 (2004)

16. Willingale, R., Kunieda, H., Okajima, T., Naitoh, M.: Optical design of the X-ray telescope for XEUS. SPIE 5900, 124 (2005)

17. Mieremet, A.L., Beijersbergen, M.W.: Fundamental spatial resolution of an X-ray pore optic. Appl. Opt. 44, 7098 (2005)

18. Treis, J., et al.: Study of noise and spectroscopic performance of DEPMOSFET matrix prototypes for XEUS. NIMA 568(1), 191 (2006)

19. Watanabe, S., et al.: Development of CdTe pixel detectors for Compton cameras. NIMA 567, 150 (2006)

20. de Korte, P., et al.: EURECA: a European-Japanese micro-calorimeter array. Proc. SPIE 6266, 62661Z (2006)

21. Barret, D., et al.: XTRA: the fast X-ray timing detector on XEUS. Proc. SPIE 5501, 23 (2004)

22. Bellazzini, R., et al.: Direct reading of charge multipliers with a self-triggering CMOS analog chip with $105 \mathrm{k}$ pixels at 50micron pitch. NIM A. 566, 552 (2006)

23. Bellazzini, R., et al.: A sealed gas pixel detector for X-ray astronomy. NIMA. 579, 853 (2007) 